\title{
Umbilical cord-derived mesenchymal stem cell therapy for neurological disorders via inhibition of mitogen-activated protein kinase pathway-mediated apoptosis
}

\author{
RUOYU ZHANG ${ }^{1}$, HUAIHONG CHEN ${ }^{1}$, ZHE ZHENG $^{2}$, QIANG LIU ${ }^{1}$ and LEI XU ${ }^{1}$ \\ Departments of ${ }^{1}$ Geriatrics and ${ }^{2}$ Neurosurgery, The Second Affiliated Hospital, School of Medicine, \\ Zhejiang University, Hangzhou, Zhejiang 310009, P.R. China
}

Received November 2, 2013; Accepted April 2, 2014

DOI: $10.3892 / \mathrm{mmr} .2014 .2985$

\begin{abstract}
The present study investigated the recovery and intrathecal administration of human umbilical cord-derived mesenchymal stem cells (HUC-MSCs) by lumbar puncture and analyzed the technical difficulties and short and long-term effects of UC-MSC transplantation in various neurological conditions. In total, 100 patients who underwent subarachnoid placement of UC-MSCs between December 2006 and May 2010 were included in the present study. The present study evaluated the number of attempts, localization of subarachnoid space and postprocedural complications. The Hauser Ambulation Index was employed for functional assessment. Clinical symptoms, the associated biochemical index and photographic examinations were observed regularly. HUC-MSCs were transplanted into mice as well as patients in order to determine the underlying therapeutic mechanisms. Technical difficulties were encountered in 31 patients $(31 \%)$ in the form of general anesthesia supplementation and difficulty localizing lumbar space. Side effects were observed in $22(22 \%)$ patients, which resolved with symptomatic treatment within $48 \mathrm{~h}$. On follow-up one year later, functional indices improved in $47(47 \%)$ patients. Transplantation of HUC-MSCs inhibited apoptosis and the protein expression of c-Jun N-terminal kinase and p38 as well as triggered the phosphorylation of P-42/44 extracellular-signal-regulated kinase. In conclusion, intrathecal administration of UC-MSCs is safe and effective with no long-term adverse effects in neurological disorders. HUC-MSCs may achieve these effects via the mitogen-activated protein kinase pathway. The results suggest that there is a promise of restoration of lost tissue and improvement
\end{abstract}

Correspondence to: Dr Ruoyu Zhang, Department of Geriatrics, The Second Affiliated Hospital, School of Medicine, Zhejiang University, 88 Jiefang Road, Hangzhou, Zhejiang 310009, P.R. China

E-mail: zhangruoyu831@yeah.net

Key words: neurological diseases, stem cell, intrathecal administration routes, lumbar puncture of function in patients with profound neurological defects. These data support expanded double blind, placebo-controlled studies for this treatment modality.

\section{Introduction}

Mesenchymal stem cells (MSCs) derived from the human umbilical cord (HUC), known as HUC-MSCs, represent a prospective cell source and hold great promise for tissue engineering and therapy $(1,2)$. Compared with bone marrow MSCs they exhibit considerable advantages including the lack of ethical controversy, accessibility, extraction procedures that are painless for donors, a reduced risk of contamination, osteogenic differentiation capacities, ex vivo expansion, faster proliferation and a higher immunomodulatory capacity (2). Furthermore, there are few ethical restrictions or medico-legal limitations on extracting and applying these cells $(2,3)$. The ample resources of the cords and the feasible cryopreservation of HUC-MSCs allow these cells to be preserved for engineering applications in the future (3). Animal experiments have demonstrated that HUC-MSCs may be useful in the treatment of neuron disease and multiple selerosis (3-6). However, the safety and efficacy of stem cell therapy depends on the mode of cell administration. Furthermore, techniques aimed at increasing the number of HUC-MSCs isolated from umbilical cords (UCs) are extremely valuable.

Previous studies have indicated that the intrathecal treatment of UC-MSCs is safe, less invasive and a more convenient procedure involving no surgery (7-10). Currently, the efficiency of UC-MSC transplantation is limited to the grafting method (7-10). However, the grafting process may decrease the viability of the UC-MSCs. Therefore, enhancing the viability of UC-MSCs is the best way to improve the efficiency of UC-MSC transplantation.

The present study speculated that inhibiting the apoptosis of UC-MSCs may enhance the viability and survival of UC-MSCs. The role of the mitogen-activated protein kinase pathway in the pathogenesis of neurological disorders was also investigated. A prospective analysis was performed to assess the safety, therapeutic effect and the technical difficulties of HUC-MSCs intrathecal infusion in patients. 


\section{Patients and methods}

Characteristics of participants. In total, 100 patients with neurological disorders were recruited between December 2006 and May 2010 from The Second Affiliated Hospital, School of Medicine,Zhejiang University (Hangzhou, Zhejiang, China). A total of 53 males and 47 females were enrolled in the present study with a male to female ratio of 1.12:1. The age at diagnosis ranged between 2 and 68 years (median 40 years). In terms of diagnosis, 30 patients had spinal cord injury, 15 patients had cerebral palsy, 15 patients had post-traumatic brain syndrome, 8 patients had post-brain infarction syndrome, 8 patients had spinocerebellar ataxias and 12 patients had motor neuron disease (Table I). The local institutional review board of The Second Affiliated Hospital of Medicine, under the auspices of the National Ministry of Health, approved application of the technique and written informed consent was obtained from each patient prior to initiation of the treatment. Patients were excluded from the present study if they met the following criteria: i) prior history of severe allergic reactions; ii) history of, or active, malignancy; iii) active systemic or severe focal infections, including HIV and syphilis; iv) active cardiac, pulmonary, renal, hepatic or gastrointestinal disease; v) coagulopathy or any other contraindication for lumbar puncture; vi) gastrostomy, tracheostomy or noninvasive ventilatory support as these can effect the prognosis and end-point measurements; vii) any severe psychiatric disorder and viii) any immunodeficiency disease or condition. As per protocol, the pre- and post-treatment study assessments included complete blood counts, routine urine tests, analysis of liver function, renal function, electrolytes, sero-enzymology, blood glucose, blood lipids, cellular and humoral immunity, routine cerebrospinal fluid (CSF) and biochemical markers (biochemistry analyzer and Epics-XL flow cytometer; Beckman Coulter, Inc. (Pasadena, CA, USA).

Characteristics of HUC-MSCs. The isolation, culture and expansion of the HUC-MSCs were performed as previously described by $\mathrm{Gu}$ et al (10). In brief, human umbilical cord (HUC) was obtained from the Gynecology Department at Renmin Hospital, Hubei University of Medicine (Shiyan, Hubei, China). Tissue collection was approved by the Ethics Committee of Renmin Hospital and informed consent was obtained from the newborns' parents. The selected tissue was sliced into $1-2 \mathrm{~mm}^{3}$ pieces and then incubated with $0.075 \%$ collagenase type II (Sigma, St. Louis, MO, USA) for $30 \mathrm{~min}$ and with $0.125 \%$ trypsin (Gibco-BRL, Grand Island, NY, USA) for $30 \mathrm{~min}$. To obtain the cell suspensions, the treated tissue was passed through a $100 \mathrm{~mm}$ filter. The low glucose Dulbecco's modified Eagle's medium (DMEM; Gibco-BRL) and $5 \%$ fetal bovine serum (FBS; HyClone, Logan, UT, USA), supplemented with $10 \mathrm{ng} / \mathrm{ml}$ epidermal growth factor (Sigma), $100 \mathrm{mg} / \mathrm{ml}$ streptomycin (Sigma), $10 \mathrm{ng} / \mathrm{ml}$ vascular endothelial growth factor (Sigma), $100 \mathrm{U} / \mathrm{ml}$ penicillin and $2 \mathrm{mmol} / 1$ glutamine (Gibco-BRL) were employed in the present study. For the cultural condition, the cells were cultured in an appropriate atmosphere at $37^{\circ} \mathrm{C}$ with $5 \%$ $\mathrm{CO}_{2}$. Once the cell monolayer was formed, flow cytometric analysis was used for the detection of CD29, CD105, CD44
Table I. Clinical conditions of the investigated patients.
Condition

Number of patients

Spinal cord injury

Cerebral palsy

Post-traumatic brain syndrome

Post-brain infarction syndrome

Spinocerebellar ataxias

Motor neuron disease

Patients with technical difficulties

General anesthesia supplementation

Taylor's approach

Multiple attempts

Side effects

Headache

Low-grade fever

Low back pain

Lower limb pain

Improvement in functional indices

Spinal cord injury

Cerebral palsy

Post-traumatic brain syndrome

Post-brain infarction syndrome

Spinocerebellar ataxias

Motor neuron disease
Number of patients

35

20

20

9

8

8

$31(31.0 \%)$

18

7

6

$22(22.0 \%)$

13

5

2

2

$47(47.0 \%)$

16

12

10

6

2

1 and CD166 positive cells and for CD34, CD14, CD45, CD38 and HLA-DR negative cells.

Cell administration. Patients' computed tomography scans and/or magnetic resonance images of the spine and brain were reviewed prior to the procedure, and the intervertebral space between lumbar vertebrae three and four was selected for HUC-MSCs placement. HUC-MSCs were administered via intrathecal injection by lumbar puncture. Each patient received cell transplantation four to six times depending on the patient's condition, within an interval of 5-7 days. CSF ( $2 \mathrm{ml}$ ) was removed and replaced by $2 \mathrm{ml}$ of cell suspension during the intrathecal injection. The needle was maintained in the same position for $5 \mathrm{~min}$ prior to withdrawal. Pediatric and uncooperative patients were administered general anesthesia prior to performing lumbar puncture. The present study evaluated the number of attempts, localization of subarachnoid space and postprocedural complications. All patients were monitored in the wards for $24 \mathrm{~h}$ and hydrated with 3 liters of fluid; ambulation was allowed $8 \mathrm{~h}$ postprocedure. Short and long term functional evaluation was performed using the Hauser Ambulation Index (HAI) by the HUC-MSCs transplant team on a regular basis. The HUC-MSCs transplant process was performed according to the study by Dalous et al (12).

Therapeutic effect. To the best of our knowledge there are no published criteria to measure therapeutic efficacy in the treat- 
Table II. Spinal cord injury clinical profile.

\begin{tabular}{|c|c|}
\hline Condition & Number \\
\hline Number of patients & 38 \\
\hline Pattern of injury & 12 \\
\hline Complete transection & $22 \%$ \\
\hline Nontransection & $78 \%$ \\
\hline Injury to treatment duration & 4 months to 2 years \\
\hline \multicolumn{2}{|l|}{ Clinical and functional benefit } \\
\hline$<1$ year & $13(35.3 \%)$ \\
\hline$>1$ year & $6(18.7 \%)$ \\
\hline \multicolumn{2}{|c|}{ Pattern of motor improvement according to $\mathrm{HAI}^{\mathrm{a}}$} \\
\hline$<1$ year & 13 \\
\hline \multicolumn{2}{|l|}{ Posttransplant grade } \\
\hline 4 & 2 \\
\hline 5 & 3 \\
\hline 6 & 2 \\
\hline 7 & 3 \\
\hline$>1$ year & 5 \\
\hline \multicolumn{2}{|l|}{ Posttransplant grade } \\
\hline 6 & 3 \\
\hline 7 & 1 \\
\hline 8 & 1 \\
\hline Autonomic improvement & 12 \\
\hline$<1$ year & 8 \\
\hline Catheter free & 2 \\
\hline Intermittent catheterization & 3 \\
\hline Bowel sensations and sweating & 2 \\
\hline$>1$ year & 4 \\
\hline Intermittent catheterization & 3 \\
\hline Improved bladder tone & 1 \\
\hline
\end{tabular}

${ }^{\text {a}}$ Fifteen patients were HAI grade 9 prior to transplantation. HAI, Hauser Ambulation Index.

ment of HUC-MSCs for neurological disorders. The HAI was applied for the evaluation of treatment efficacy (11). The HAI is a rating scale developed by Hauser et al (11) to assess mobility by evaluating the time and degree of assistance required to walk 25 feet. The scores ranged between 0 (asymptomatic and fully active) and 10 (bedridden) as follows: 0 = asymptomatic, fully active; 1 = walks normally, however, reports fatigue that interferes with athletic or other demanding activities; $2=$ abnormal gait or episodic imbalance, gait disorder is noticed by family and friends, able to walk 25 feet ( 8 meters) in $\leq 10 \mathrm{sec} ; 3=$ walks independently, able to walk 25 feet in $\leq 20 \mathrm{sec}$; $4=$ requires unilateral support (cane or single crutch) to walk, walks 25 feet in $\leq 20 \mathrm{sec} ; 5=$ requires bilateral support (canes, crutches or walker) and walks 25 feet in $\leq 25 \mathrm{sec}$ or requires unilateral support but requires $>20 \mathrm{sec}$ to walk 25 feet; $6=$ requires bilateral support and $>20 \mathrm{sec}$ to walk 25 feet, may use wheelchair on occasion; 7 = walking limited to several steps with bilateral support, unable to walk 25 feet, may use wheelchair for the majority of activities; $8=$ restricted to wheelchair, able to transfer self independently; 9 = restricted to wheelchair, unable to transfer self independently; $10=$ bedridden.

\section{Results}

Autonomic improvement of spinal cord injury. Administration of HUC-MSCs via intrathecal routes was well tolerated during the clinical treatment course. Out of 88 patients, technical difficulties were encountered in 20 patients (23\%), 12 of which required general anesthesia supplementation, three required Taylor's approach and five required multiple attempts for the localization of subarachnoid space. In total, 10 patients suffered from postprocedural headache, which was relieved within $24 \mathrm{~h}$ with analgesics, hydration and rest; 3 patients had low-grade fever lasting for $24 \mathrm{~h} ; 3$ patients had lower back pain and 2 patients had lower limb pain, which was responded to within $24 \mathrm{~h}$ of symptomatic treatment. On long-term follow-up, functional indices improved in $50(31.67 \%)$ patients, including 15 patients with spinal cord injury, 10 with cerebral palsy, 10 with post-traumatic brain syndrome, 5 with post-brain infarction syndrome, 5 with spinocerebellar ataxias and 5 with motor neuron disease. Patients with cerebral palsy and post-traumatic brain syndrome demonstrated improvement in muscle tone, rigidity and spasm (Table I). 


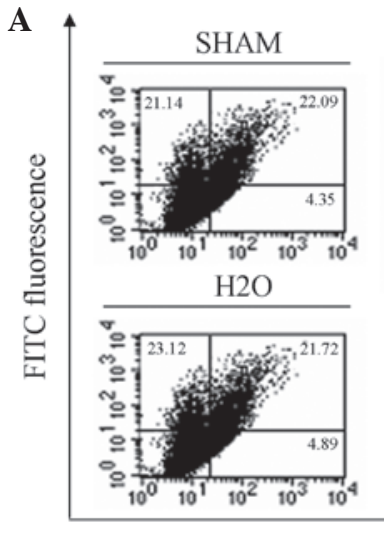

B
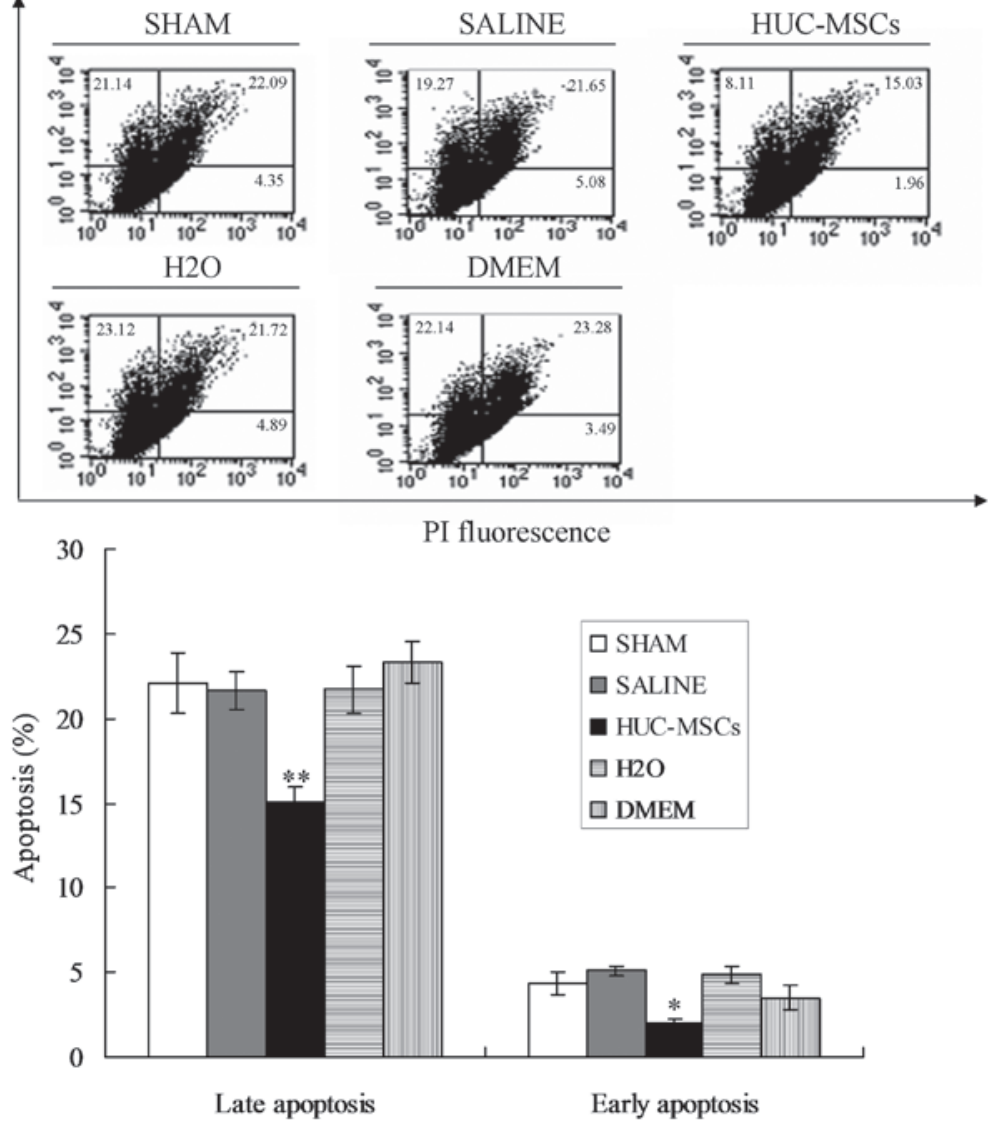

Figure 1. Apoptosis was observed in UC-MSC-treated mice. (A) Annexin V/PI double staining assays of cells. (B) Statistical analysis. The X axis indicates the number of PI stained cells. The Y axis indicates the number of Annexin V-FITC stained cells. The percentage of the cells labeled with only Annexin V (counted in Q1 region) in each preparation are shown in each figure. The results from three independent experiments are shown. The UC-MSC-treated group against the SHAM group are presented as the mean \pm standard deviation and illustrated as ${ }^{*} \mathrm{P}<0.05$ and ${ }^{* *} \mathrm{P}<0.01$, respectively. HUC-MSCs, human umbilical cord mesenchymal stem cells; FITC, fluorescein isothiocyanate; DMEM, Dulbecco's modified Eagle's medium; PI, propidium iodide.

Out of the 30 spinal cord injury patients, 20 had a previous history of spinal surgery. In 15 spinal cord injury patients with HAI scale improvement, 10 patients had an injury period of $<1$ year, whereas 5 had an injury period of $>1$ year. A total of 15 patients showed improvement in motor power (10 with an injury period of $<1$ year and $5>1$ year). Prior to treatment, all these patients were HAI grade 9. Following HUC-MSC transplantation, one was grade 4, two were grade 5, three were grade 6 and four were grade 7 . A marked improvement was observed in four bedridden patients who were able to walk with the help of a walker (HAI grade 9-HAI grade 4/5). In 12 patients, autonomic improvement was observed, including 8 patients with an injury period of $<1$ year. Of these, three became catheter-free and two required intermittent catheterization. In addition, 3 patients showed improvement in bowel sensations and sweating. A mixed motor and autonomic improvement was observed in 8 of 30 patients (Table II).

HUC-MSC transplantation inhibits apoptosis. In order to investigate the therapeutic effect of HUC-MSCs on neurological disorders, HUC-MSCs were transplanted into mice. The results indicated that HUC-MSCs were able to significantly inhibit apoptosis of neurocytes compared with the other groups (Fig. 1; $\mathrm{P}<0.01$ ).
HUC-MSCs inhibit apoptosis via the mitogen-activated protein kinase (MAPK) and extracellular-signal-regulated kinase (ERK)1/2 pathways. The activation of the other two members of MAPKs, c-Jun N-terminal kinase and p38, decreased the rate of apoptosis following treatment with HUC-MSCs (Fig. 2). On the basis of these observations, the effect of normothermic ischemia-reperfusion stress on the different members of the MAPK family were investigated. The expression of phospho-p 44/42 ERK1/2 was significantly higher in the liver of animals treated with HUC-MSCs (Fig. 3).

\section{Discussion}

Mesenchymal stem cells (MSCs) are highly proliferative in vitro, have multilineage differentiation potential and are able to be obtained from various tissue sources, including the bone marrow (BM), adipose tissue and umbilical cord blood. The BM is the main source for isolation of MSCs and BM-derived MSCs (BM-MSCs) have been studied extensively. However, BM harvesting is a highly invasive method for the donors. Furthermore, the multipotent differentiation potential, maximal lifespan of BM-MSCs and proliferation efficiency are correlated with the age of the patients. In addition, MSCs can be isolated from umbilical cord Wharton's jelly and differentiate into different types of cells, including adipocytes, osteocytes, 
A

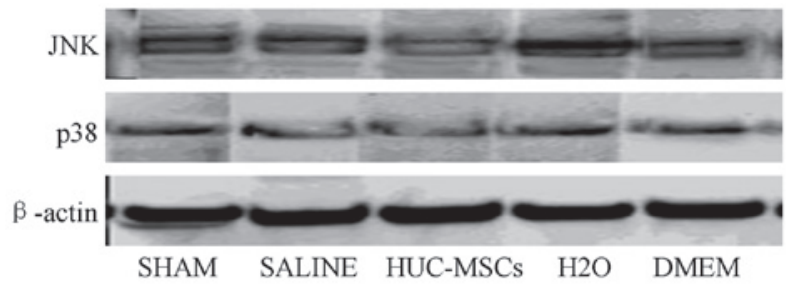

B

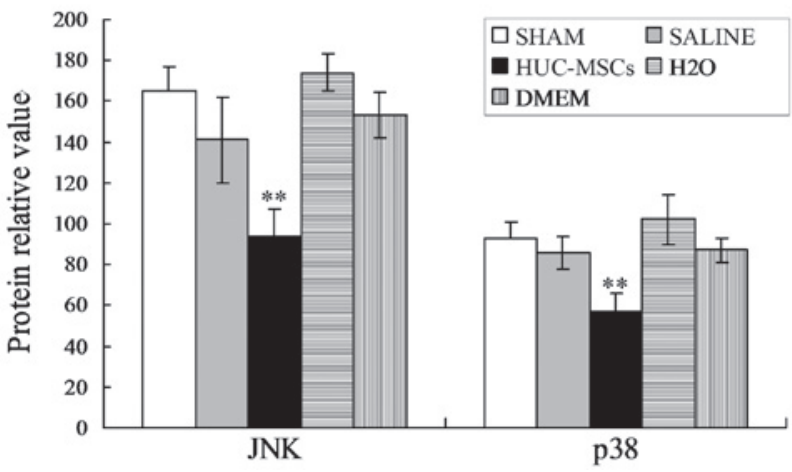

Figure 2. HUC-MSC transplantation results in a decrease of JNK and p38 in mice. (A) Western blot analysis; (B) statistical analysis. The UC-MSC-treated group compared with the SHAM group are presented as the mean \pm standard deviation and illustrated as ${ }^{* *} \mathrm{P}<0.01$, respectively. HUC-MSCs, human umbilical cord mesenchymal stem cells; DMEM, Dulbecco's modified Eagle's medium; JNK, c-Jun N-terminal kinase.

A

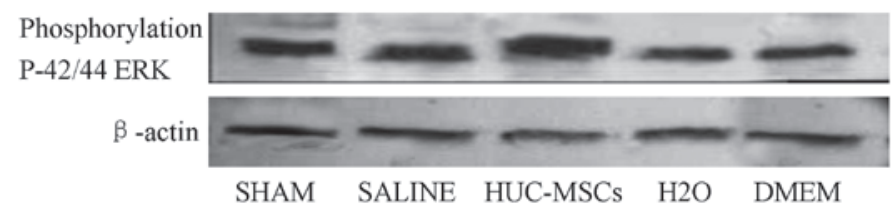

B

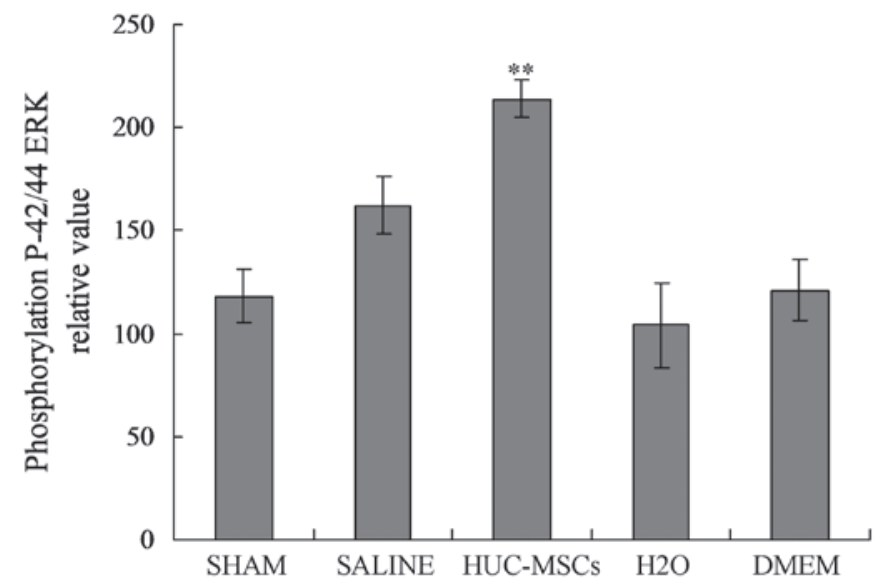

Figure 3. P-42/44 ERK phosphorylation may be involved in the inhibition of apoptosis in UC-MSC-transplanted mice. (A) Western blot analysis; (B) statistical analysis. The UC-MSC-treated group compared with the SHAM group are presented as the mean \pm standard deviation and illustrated as ${ }^{* *} \mathrm{P}<0.01$, respectively. UC-MSCs, umbilical cord mesenchymal stem cells; ERK, extracellular-signal-regulated kinase; DMEM, Dulbecco's modified Eagle's medium.

chondrocytes and neurons. Compared with the MSCs isolated from other tissues, UC-MSCs can differentiate into many types of cell, including blood, nerve and bone cells. Furthermore, this type of MSC does not express the major histocompatibility complex (MHC) class II (HLA-DR) antigens (13), which is expressed in other types of MSCs. Several studies have indicated that UC-MSCs retain their viable status, and are not rejected even four months following transplantation. Therefore, immune suppression is unnecessary for UC-MSCs transplantation, which suggests that UC-MSCs are a feasible and stable cell source for transplantation. Notably, the umbilical cord can be easily obtained and isolated. Thus, UC-MSCs transplantation may act as a potential MSC cell source for tissue engineering.

Several studies have demonstrated that the transplantation of UC-MSCs is a potential therapeutic strategy for the treatment of central nervous system diseases, including cerebrovascular disease, nerve degenerative diseases, spinal cord injury and cerebral palsy. The transplantation path in cell therapy is diverse (14-16) and mainly includes the intervention path, local implantation, intravenous route and lumbar puncture way. Migration toward pathology is the first critical step in UC-MSCs engagement during regeneration and it is hypothesized that the 
inflammatory response itself guides the behavior of potentially reparative UC-MSCs. It has been revealed that introducing UC-MSCs into the subarachnoid space of the spinal cord transports the cells through CSF and allows more efficient delivery of cells to the injured area of the central nervous system compared with the intravenous route.

Intrathecal administration of UC-MSCs is safe, less invasive and a more convenient procedure involving no surgery. However, the efficacy of UC-MSCs transplantation is limited by the grafting method. The mechanical process of grafting may decrease the viability and survival of the transplanted MSCs. Therefore, for the transplantation of MSCs it is necessary to improve the viability and survival of UC-MSCs prior to transplantation (14). The present study examined the viability of MSCs using the trypan blue exclusion assay. The patient should be positioned to ensure the intervertebral spaces are detectable for lumbar puncture for transplantation of cells into the CSF.

At present, the optimized method for injecting cells is to slowly deliver a fixed volume at a constant rate. The quantity and density of transplanted MSCs are critical to ensure an adequate number of cells for grafting and optimal survival. For the injection procedure, the needle tip should be left in the same position after the cells have been injected.

Notably, UC-MSCs cell transplantation was able to inhibit apoptosis (Fig. ). It is well established that cell apoptosis can trigger neurological diseases and degenerative diseases. In the present study, UC-MSCs improved the disease status through inhibiting the apoptotic process of the cells.

In the present study, a significant number of patients with spinal cord injury were included. A lumbar puncture was performed in these patients, however, this was technically difficult (17-20) due to the following reasons: pathological scoliosis and positional difficulties. Certain other factors, including cognitive, behavioral and communication problems as well as coexisting diseases and the specific drug therapy methods, affected the anesthetic management. In addition, other problems affected the transplantation of MSCs, including gastroesophageal reflux, electrolyte imbalance and pulmonary aspiration in the present study. Furthermore, the positioning of the patients for the transplantation was difficult as analgesia and inadequate anesthesia may lead to increased muscle tone and spasm. Thus, judicious use of an anesthetic agent intraoperatively was critical to ensure a relaxed peri-operative and post-operative period. Following intraspinal injection of UC-MSCs in this patient population, certain patients $(n=13)$ suffered from postprocedural headache. This was possibly due to numerous reasons, including the alteration in CSF circulation, leakage of CSF and the use of a large-bore spinal needle, which was essential to prevent any mechanical damage to the cells during infusion. Furthermore, 3 patients suffered from lower back pain and 2 patients had lower limb pain, possibly due to nerve root injuries; however, they recovered quickly. All the side effects resolved within $24 \mathrm{~h}$ with no long-term sequelae.

In conclusion, the present study indicated that subarachnoid transplantation with UC-MSCs in neurological disorders may be a promising therapy, which is relatively safe, simple to perform and has no long-term adverse effects. However, certain studies have provided different conclusions, and thus this method requires further investigation so that the potential of this therapy may be fully realized.

\section{Acknowledgements}

The authors would like to thank the Professional associates for their assistance in obtaining patient data throughout the present study. This study was supported by Zhejiang Provincial Qianjiang talent plan (grant no. 2012R10041), China National Funds for Young Scientists (grant no. 81100241), the Zhejiang Provincial Natural Science Foundation (grant no. Y2110033), The Ph.D. Programs Foundation of the Ministry of Education of China (grant no. 20110101120119), the Science and Technology Department of Zhejiang Province Public Technology Research and the social development project (grant no. 2013C33131).

\section{References}

1. Wang L, et al: Protective effect of transplanted bone marrow-derived mesenchymal stem cells on pancreatitis-associated lung injury in rats. Mol Med Rep 6: 287-292, 2012.

2. Trivanović D, Kocić J, Mojsilović S, et al: Mesenchymal stem cells isolated from peripheral blood and umbilical cord Wharton's jelly. Srp Arh Celok Lek 41: 178-186, 2013.

3. Titomanlio L, et al: Stem cell therapy for neonatal brain injury: perspectives and challenges. Ann Neurol 70: 698-712, 2011.

4. Liu J, Han D, Wang Z, et al: Clinical analysis of the treatment of spinal cord injury with umbilical cord mesenchymal stem cells. Cytotherapy 15: 185-191, 2013.

5. Wang L, Ji H, Zhou J, et al: Therapeutic potential of umbilical cord mesenchymal stromal cells transplantation for cerebral palsy: a case report. Case Rep Transplant 2013: 146347, 2013.

6. O'Hare E and Young PJ: Childhood spinal muscular atrophy and stem cell research: Is cellular replacement therapy the answer? (Review). Mol Med Rep 2: 3-5, 2009.

7. Lim JY, Jeong CH, et al: Therapeutic effects of human umbilical cord blood-derived mesenchymal stem cells after intrathecal administration by lumbar puncture in a rat model of cerebral ischemia. Stem Cell Res Ther 2: 38, 2011.

8. Kang EJ, Lee YH, Kim MJ, et al: Transplantation of porcine umbilical cord matrix mesenchymal stem cells in a mouse model of Parkinson's disease. J Tissue Eng Regen Med 7: 169-182, 2013.

9. Zhang XJ, Luan ZG and Ma XC: shRNAs targeting high-mobility group box-1 inhibit E-selectin expression via homeobox A9 in human umbilical vein endothelial cells. Mol Med Rep 7: 1251-1256, 2013.

10. Gu Z, Akiyama K, Ma X, et al: Transplantation of umbilical cord mesenchymal stem cells alleviates lupus nephritis in MRL/lpr mice. Lupus 19: 1502-1514, 2010.

11. Hauser SL, Dawson DM, et al: Intensive immunosuppression in progressive multiple sclerosis. A randomized, three-arm study of high-dose intravenous cyclophosphamide, plasma exchange, and ACTH. N Engl J Med 308: 173-180, 1983.

12. Dalous J, Larghero J and Baud O: Transplantation of umbilical cordderived mesenchymal stem cells as a novel strategy to protect the central nervous system: technical aspects, preclinical studies, and clinical perpectives. Pediatr Res 71: 482-490, 2012.

13. Glass JD, Boulis NM, et al: Lumbar intraspinal injection of neural stem cells in patients with amyotrophic lateral sclerosis: results of a phase I trial in 12 patients. Stem Cells 30: 1144-1151, 2012.

14. Wang H, Yang Y, et al: Programming of human umbilical cord mesenchymal stem cells in vitro to promote pancreatic gene expression. Mol Med Rep 8: 769-774, 2013.

15. Lepore AC: Intraspinal cell transplantation for targeting cervical ventral horn in amyotrophic lateral sclerosis and traumatic spinal cord injury. J Vis Exp: Sep 18, 2011. doi: 10.3791/3069.

16. Wiley CA and Achim C: Human immunodeficiency virus encephalitis is the pathological correlate of dementia in acquired immunodeficiency syndrome. Ann Neurol 36: 673-676, 1994.

17. Xu W, et al: Chitooligosaccharides and N-acetyl-D-glucosamine stimulate peripheral blood mononuclear cell-mediated antitumor immune responses. Mol Med Rep 6: 385-390, 2012.

18. Ciardi A, et al: The involvement of the cerebral cortex in human immunodeficiency virus encephalopathy: a morphological and immunohistochemical study. Acta Neuropathol 81: 51-59, 1990.

19. Cursio R, Gugenheim J, Ricci JE, et al: A caspase inhibitor fully protects rats against lethal normothermic liver ischemia by inhibition of liver apoptosis. FASEB J 13: 253-261, 1999. 